\title{
A New Strategy in Inhibition of Cathepsin B, A Target in Tumor Invasion and Metastasis
}

\author{
In Taek Lim, Samy O. Meroueh, Mijoon Lee, Mary Jane Heeg, and Shahriar Mobashery* \\ Department of Chemistry and Biochemistry, University of Notre Dame, Notre Dame, IN 46556, USA. \\ Department of Chemistry, Wayne State University, Detroit, MI 48202, USA
}

\section{Supporting Information}

Compound $\mathbf{1 5}$ was prepared in $11 \%$ yield by the reaction of $\mathbf{1 4}$ with an activated form of $\mathbf{1 1}$ by treatment of oxalyl chloride. The synthesis of compound 16, as depicted in Scheme S1, starting with compound 18, which itself was prepared according to a known procedure. ${ }^{1}$ Removal of the chiral auxiliary gave 19, which was then transformed to $\mathbf{2 0}$ by coupling with L-proline benzyl ester. Selective deprotection of the tert-butyl group of 20 by treatment with TFA afforded 21. Compound 16 was obtained by the coupling of hydroxamic acid $\mathbf{1 4}$ and $\mathbf{2 1}$ using the PYBOP reagent.

\section{$\underline{\text { Scheme S1 }}$}

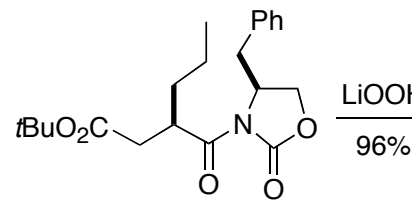

18<smiles>CCCOC(=O)CC(CCC)C(=O)O</smiles>

19<smiles>CCCC(CC(=O)OCc1ccccc1)C(=O)N1CCCC1C(=O)OCc1ccccc1</smiles>

20

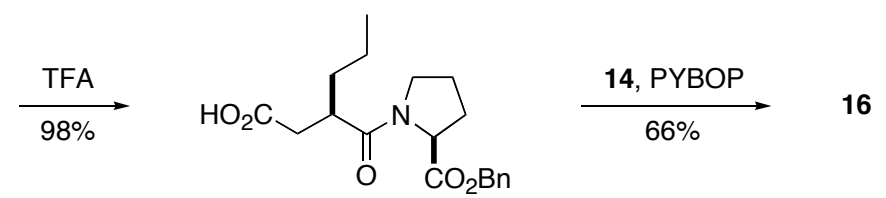

21

Compound 17 was synthesized according to Scheme S2. The commercially available phenylalanine chloromethyl ketone $\mathbf{2 2}^{2}$ was allowed to react with difluorobenzoyl chloride to give $\mathbf{2 3}$. Compound $\mathbf{2 3}$ was 
converted to acetoxymethyl ketone $\mathbf{2 4}$, which was transformed to hydroxymethyl ketone $\mathbf{2 5}$ by the reaction of potassium cyanide. A PYBOP-mediated reaction of $\mathbf{1 1}$ and $\mathbf{2 5}$ furnished the desired compound $\mathbf{1 7 .}$

$\underline{\text { Scheme S2 }}$
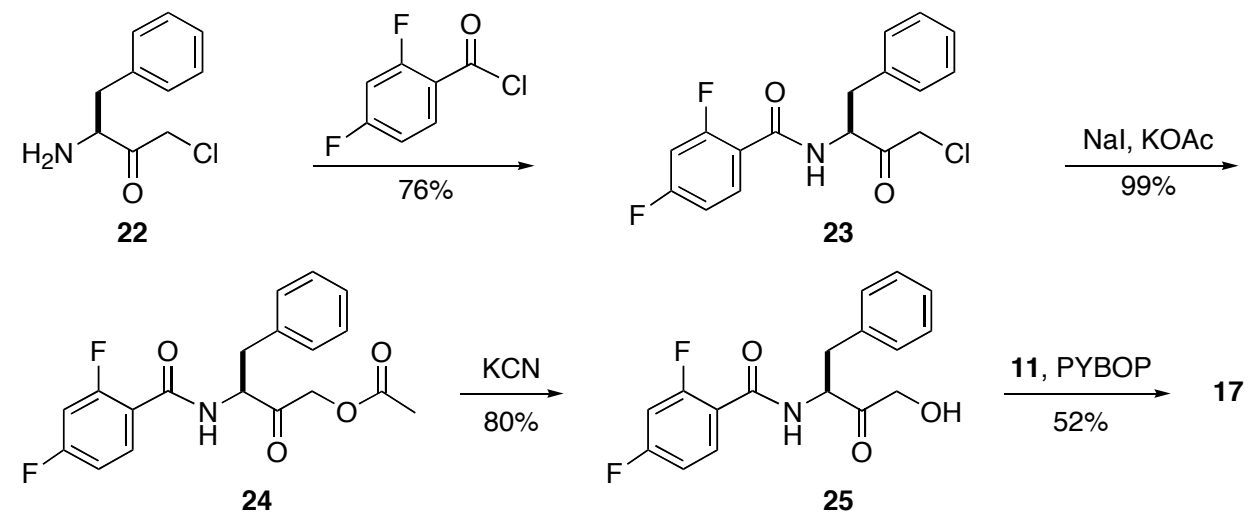

\section{1-(2(R)-\{1(S)-Hydroxy-2-[(S)-2,4-difluorobenzoyl-phenylalanylaminooxy]-2-oxo-ethyl $\}-$}

pentanoyl)-proline benzyl ester (15). A solution of oxalyl chloride ( $2 \mathrm{M}$ in $\left.\mathrm{CH}_{2} \mathrm{Cl}_{2}, 0.5 \mathrm{~mL}, 1.0 \mathrm{mmol}\right)$ in dichloromethane was added to a solution of compound 11 (368 mg, $0.91 \mathrm{mmol})$ in dichloromethane (3 $\mathrm{mL}$ ) in the presence of $30 \mu \mathrm{L}$ of DMF at $-5{ }^{\circ} \mathrm{C}$. After $2 \mathrm{~h}$, the solvent was removed under reduced pressure, while the distillation flask was kept in an ice-water bath. The residue was dissolved in dichloromethane $(2 \mathrm{~mL})$ and added to a solution of hydroxamide 14 (291 $\mathrm{mg}, 0.91 \mathrm{mmol})$ and pyridine (73 $\mu \mathrm{L}, 0.91 \mathrm{mmol})$ in dichloromethane $(3 \mathrm{~mL})$ in an ice-water bath. After an additional $2 \mathrm{~h}$, the solvent was evaporated under reduced pressure. Column chromatography $\left(\mathrm{CHCl}_{3}: \mathrm{EtOH}: \mathrm{AcOH}=60: 1: 0.2\right)$ at $4{ }^{\circ} \mathrm{C}$ afforded the title compound (64 mg, 11\%); ${ }^{1} \mathrm{H}$ NMR $\left(500 \mathrm{MHz}, \mathrm{CDCl}_{3}\right) \delta 8.09(\mathrm{~m}, 1 \mathrm{H}), 7.32-7.18$ (m, 12H), $6.98(\mathrm{~m}, 1 \mathrm{H}), 6.83(\mathrm{~m}, 1 \mathrm{H}), 5.29$ (q, $\left.J=7.8 \mathrm{~Hz}, 2 \mathrm{H}, \mathrm{OC}_{2} \mathrm{Ph}\right), 5.06\left(\mathrm{~m}, 1 \mathrm{H}, \mathrm{CHCH}_{2} \mathrm{Ph}\right), 4.61$ (dd, $J$ $\left.=8.5,4.5 \mathrm{~Hz}, 1 \mathrm{H}, \underline{\mathrm{HCO}}_{2} \mathrm{Bn}\right), 4.20(\mathrm{dd}, J=12.0,5.5 \mathrm{~Hz}, 1 \mathrm{H}, \mathrm{C} \underline{\mathrm{HOH}}), 3.71\left(\mathrm{~m}, 2 \mathrm{H}, \underline{\mathrm{C}}_{2} \mathrm{NR}_{2}\right), 3.33(\mathrm{dd}, J$ $\left.=14.0,6.5 \mathrm{~Hz}, 1 \mathrm{H}, \mathrm{CHCH}_{2} \mathrm{Ph}\right), 3.22\left(\mathrm{dd}, J=14.5,8.5 \mathrm{~Hz}, 1 \mathrm{H}, \mathrm{CHC}_{2} \mathrm{Ph}\right), 3.14(\mathrm{~m}, 1 \mathrm{H}, \mathrm{C} \underline{\mathrm{HPr}}), 2.21(\mathrm{~m}$, 1H), 2.11-1.98 (m, 4H), $1.75\left(\mathrm{~m}, 2 \mathrm{H}, \underline{\mathrm{CH}}_{2} \mathrm{Et}\right), 1.48\left(\mathrm{~m}, 1 \mathrm{H}, \mathrm{C}_{2} \mathrm{CH}_{3}\right), 1.27\left(\mathrm{~m}, 1 \mathrm{H}, \mathrm{CH}_{2} \mathrm{CH}_{3}\right), 0.86(\mathrm{t}, J=$ $\left.7.3 \mathrm{~Hz}, 3 \mathrm{H}, \mathrm{CH}_{2} \mathrm{CH}_{3}\right) ;{ }^{13} \mathrm{C} \mathrm{NMR}\left(125 \mathrm{MHz}, \mathrm{CDCl}_{3}\right)$ d 174.9, 174.4, 171.4, 168.6, 165.4 (dd, $J=256.2$, $12.1 \mathrm{~Hz}, \underline{\mathrm{CF}}), 163.3,161.3(\mathrm{dd}, J=251.6,12.9 \mathrm{~Hz}, \underline{\mathrm{CF}}), 135.9,135.8,133.9,129.5,128.9,128.8,128.5$, 128.4, 127.4, 116.6, $112.6(\mathrm{~d}, J=21.4 \mathrm{~Hz}), 104.6$ (dd, $J=28.8,26.0 \mathrm{~Hz}), 73.4,67.1,59.3,52.7$, 47.7, 44.9, 37.1, 30.0, 29.4, 25.1, 20.4, 14.3; MS (FAB) calcd for $\mathrm{C}_{37} \mathrm{H}_{39} \mathrm{~F}_{2} \mathrm{~N}_{3} \mathrm{O}_{9}\left(\mathrm{M}^{+}\right)$665.3, found 665.6.

2(R)-Propylsuccinic acid 4-tert-butyl ester (19). The reported method of Simoneau and co-workers ${ }^{1}$ was applied to remove the chiral auxiliary from 3-(4-benzyl-2-oxo-oxazolidine-3-carbonyl)-hexanoic acid 
t-butyl ester 18. 30\% Hydrogen peroxide $(5.72 \mathrm{~mL}, 60 \mathrm{mmol})$ was added to an ice-cold solution of 18 (4.97 g, $13.2 \mathrm{mmol})$ in THF: $\mathrm{H}_{2} \mathrm{O}(3: 1,120 \mathrm{~mL})$, followed by addition of $\mathrm{LiOH} \cdot \mathrm{H}_{2} \mathrm{O}(1.11 \mathrm{~g}, 26.5 \mathrm{mmol})$. The mixture was allowed to warm to room temperature and stirred for $12 \mathrm{~h}$. The mixture was cooled to icewater temperature and was treated with $1.5 \mathrm{M}$ aqueous $\mathrm{Na}_{2} \mathrm{SO}_{3}(10 \mathrm{~mL}, 15 \mathrm{mmol})$ to destroy the excess peroxide. THF was removed under reduced pressure. The residue was diluted with water $(50 \mathrm{~mL})$ and saturated aqueous $\mathrm{NaHCO}_{3}(50 \mathrm{~mL})$ and the resulting solution was washed with dichloromethane $(50 \mathrm{~mL}$ $\times 3)$. The aqueous layer was rendered acidic $(\mathrm{pH} 3)$ with $10 \%$ aqueous citric acid and the product was extracted into EtOAc $(100 \mathrm{~mL} \times 3)$. The combined organic layers were washed with brine, dried over $\mathrm{MgSO}_{4}$, filtered and concentrated under reduced pressure to give 19 (2.75 g, 96\%) as an oil; ${ }^{1} \mathrm{H}$ NMR

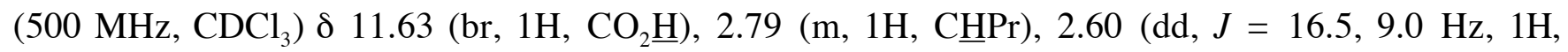
$\left.\mathrm{C}_{2} \mathrm{CO}_{2} t \mathrm{Bu}\right), 2.36\left(\mathrm{dd}, J=16.5,5.5 \mathrm{~Hz}, 1 \mathrm{H}, \mathrm{C}_{2} \mathrm{CO}_{2} t \mathrm{Bu}\right), 1.63\left(\mathrm{~m}, 1 \mathrm{H}, \mathrm{C}_{2} \mathrm{Et}\right), 1.48\left(\mathrm{~m}, 1 \mathrm{H}, \mathrm{C} \underline{\mathrm{H}}_{2} \mathrm{Et}\right)$, $1.41\left(\mathrm{~s}, 9 \mathrm{H}, \mathrm{C}\left(\mathrm{C}_{3}\right)_{3}\right), 1.36\left(\mathrm{~m}, 2 \mathrm{H}, \mathrm{C}_{2} \mathrm{CH}_{3}\right), 0.90\left(\mathrm{t}, J=7.3 \mathrm{~Hz}, 3 \mathrm{H}, \mathrm{CH}_{2} \underline{\mathrm{CH}}_{3}\right) ;{ }^{13} \mathrm{C} \mathrm{NMR}(125 \mathrm{MHz}$, $\left.\mathrm{CDCl}_{3}\right) \delta 181.9,171.4,81.2,41.4,37.3,34.0,28.2,20.3,14.1$.

1-(2(R)-tert-Butoxycarbonylmethyl-pentanoyl)-proline benzyl ester (20). The acid 19 (2.72 g, $12.6 \mathrm{mmol})$, PYBOP (5.50 g, $10.6 \mathrm{mmol})$ and 1-hydroxy-7-azabenzotriazole (1.44 g, $10.6 \mathrm{mmol})$ were dissolved in anhydrous DMF (25 mL). DIPEA $(2.64 \mathrm{~mL}, 15.1 \mathrm{mmol})$ was added dropwise to the reaction mixture at $-10^{\circ} \mathrm{C}$, followed by addition of $\mathrm{L}-\mathrm{ProOBn} \cdot \mathrm{HCl}(2.74 \mathrm{~g}, 11.3 \mathrm{mmol})$. The reaction mixture was stirred at $4{ }^{\circ} \mathrm{C}$ for $20 \mathrm{~min}$ and an additional amount of DIPEA (4.38 mL, $25.2 \mathrm{mmol}$ ) was added portionwise to the mixture. After being stirred at room temperature for $12 \mathrm{~h}$, the residue was diluted with EtOAc $(40 \mathrm{~mL})$ and was washed with brine and saturated $\mathrm{NaHCO}_{3}$. The organic layer was dried over $\mathrm{MgSO}_{4}$, filtered, and concentrated. The residue was purified on column chromatograph (hexane:EtOAc = $3: 1 \sim 2: 1)$ to afford $20(3.3 \mathrm{~g}, 77 \%) ;{ }^{1} \mathrm{H} \operatorname{NMR}\left(500 \mathrm{MHz}, \mathrm{CDCl}_{3}\right) \delta 7.32\left(\mathrm{~m}, 5 \mathrm{H}, \mathrm{C}_{6} \underline{\mathrm{H}}_{5}\right), 5.13(\mathrm{dd}, J=$ 44.5, $\left.12.0 \mathrm{~Hz}, 2 \mathrm{H}, \mathrm{OC}_{2} \mathrm{Ph}\right), 4.55\left(\mathrm{dd}, J=8.5,4.0 \mathrm{~Hz}, 1 \mathrm{H}, \underline{\mathrm{CHCO}}_{2} \mathrm{Bn}\right), 3.79\left(\mathrm{~m}, 1 \mathrm{H}, \mathrm{C}_{2} \mathrm{NR}_{2}\right), 3.66(\mathrm{~m}$, $\left.1 \mathrm{H}, \underline{\mathrm{C}}_{2} \mathrm{NR}_{2}\right), 2.91\left(\mathrm{~m}, 1 \mathrm{H}, \mathrm{C}^{\mathrm{HCONR}}{ }_{2}\right), 2.73\left(\mathrm{dd}, J=16.5,10.0 \mathrm{~Hz}, 1 \mathrm{H}, \mathrm{C}_{2} \mathrm{CO}_{2} t \mathrm{Bu}\right), 2.29(\mathrm{dd}, J=16.5$, $\left.4.0 \mathrm{~Hz}, 1 \mathrm{H}, \mathrm{CH}_{2} \mathrm{CO}_{2} t \mathrm{Bu}\right), 2.19(\mathrm{~m}, 1 \mathrm{H}), 1.98(\mathrm{~m}, 3 \mathrm{H}), 1.58(\mathrm{~m}, 1 \mathrm{H}), 1.39\left(\mathrm{~s}, 9 \mathrm{H}, \mathrm{C}\left(\mathrm{CH}_{3}\right)_{3}\right), 1.36(\mathrm{~m}, 3 \mathrm{H})$, $0.85\left(\mathrm{t}, J=7.3 \mathrm{~Hz}, 3 \mathrm{H}, \mathrm{CH}_{2} \mathrm{CH}_{3}\right) ;{ }^{13} \mathrm{C} \mathrm{NMR}\left(125 \mathrm{MHz}, \mathrm{CDCl}_{3}\right) \delta 174.3,172.5,172.2,136.0,128.7$, $128.4,128.4,80.6,66.9,59.0,47.2,39.5,38.0,35.0,29.4,28.3,25.1,20.4,14.4$; HRMS (EI) calcd for $\mathrm{C}_{23} \mathrm{H}_{33} \mathrm{NO}_{5}\left(\mathrm{M}^{+}\right)$403.23587, found 403.23631. 
1-(2(R)-Carbonylmethyl-pentanoyl)-proline benzyl ester (21). The $t$-butyl ester 20 (2.95 g, 7.3 mmol) was dissolved in dry dichloromethane $(20 \mathrm{~mL})$. A $10 \mathrm{~mL}$ portion of TFA was added dropwise at 0 ${ }^{\circ} \mathrm{C}$. The reaction mixture was stirred at room temperature for $6 \mathrm{~h}$, at which time it was concentrated under reduced pressure. The residue was dissolved in EtOAc $(20 \mathrm{~mL})$ and the solvent was evaporated in vacuum to give 21 (2.5 g, 98\%) as an oil; ${ }^{1} \mathrm{H}$ NMR (500 MHz, $\left.\mathrm{CDCl}_{3}\right) \delta 11.48\left(\mathrm{~s}, 1 \mathrm{H}, \mathrm{CO}_{2} \mathrm{H}\right), 7.34\left(\mathrm{~m}, 5 \mathrm{H}, \mathrm{C}_{6} \underline{\mathrm{H}}_{5}\right)$, $5.15\left(\mathrm{q}, J=19.5,12.0 \mathrm{~Hz}, 2 \mathrm{H}, \mathrm{OC}_{2} \mathrm{Ph}\right), 4.61\left(\mathrm{dd}, J=9.0,4.0 \mathrm{~Hz}, 1 \mathrm{H}, \mathrm{C}_{\underline{H C O}} \mathrm{Bn}\right), 3.77(\mathrm{~m}, 1 \mathrm{H}$, $\left.\mathrm{C}_{2} \mathrm{NR}_{2}\right), 3.68\left(\mathrm{~m}, 1 \mathrm{H}, \underline{\mathrm{CH}}_{2} \mathrm{NR}_{2}\right), 2.98\left(\mathrm{~m}, 1 \mathrm{H}, \mathrm{CHCONR}_{2}\right), 2.88\left(\mathrm{dd}, J=17.0,9.5 \mathrm{~Hz}, 1 \mathrm{H}, \mathrm{CH}_{2} \mathrm{CO}_{2} \mathrm{H}\right)$, $2.55\left(\mathrm{dd}, J=17.0,3.5 \mathrm{~Hz}, 1 \mathrm{H}, \underline{\mathrm{C}}_{2} \mathrm{CO}_{2} \mathrm{H}\right), 2.34(\mathrm{~m}, 1 \mathrm{H}), 2.03(\mathrm{~m}, 3 \mathrm{H}), 1.60\left(\mathrm{~m}, 1 \mathrm{H}, \underline{\mathrm{H}}_{2} \mathrm{Et}\right), 1.44(\mathrm{~m}, 1 \mathrm{H}$, $\left.\mathrm{C}_{2} \mathrm{Et}\right), 1.38\left(\mathrm{~m}, 2 \mathrm{H}, \underline{\mathrm{C}}_{2} \mathrm{CH}_{3}\right), 0.87\left(\mathrm{t}, J=7.3 \mathrm{~Hz}, 3 \mathrm{H}, \mathrm{CH}_{2} \underline{\mathrm{C}}_{3}\right) ;{ }^{13} \mathrm{C} \mathrm{NMR}\left(125 \mathrm{MHz}, \mathrm{CDCl}_{3}\right) \delta 177.6$, 176.0, 171.7, 135.5, 128.8, 128.7, 128.5, 67.5, 59.6, 48.0, 39.3, 36.2, 34.2, 29.3, 24.9, 20.3, 14.2; HRMS (EI) calcd for $\mathrm{C}_{19} \mathrm{H}_{26} \mathrm{NO}_{5}\left(\mathrm{MH}^{+}\right)$348.18110, found 348.18143; HRMS (EI) calcd for $\mathrm{C}_{19} \mathrm{H}_{25} \mathrm{NO}_{5}\left(\mathrm{M}^{+}\right)$ 347.17327, found 347.17403 .

\section{1-(2(R)-\{2-[(S)-2,4-Difluorobenzoyl-phenylalanylaminooxy]-2-oxo-ethyl $\}$-pentanoyl)-proline}

benzyl ester (16). Diisopropylethylamine $(157 \mathrm{~mL}, 0.90 \mathrm{mmol})$ was added portionwise to a mixed solution of acid 21 (174 mg, $0.50 \mathrm{mmol})$, hydroxyamide 14 (160 mg, $0.50 \mathrm{mmol})$, and PYBOP (234 mg, $0.45 \mathrm{mmol})$ in DMF $(2 \mathrm{~mL})$ at $-10{ }^{\circ} \mathrm{C}$. The resultant solution was stirred at $4{ }^{\circ} \mathrm{C}$ overnight. An additional amount of diisopropylethylamine $(26 \mathrm{~mL}, 0.15 \mathrm{mmol})$ was added to the reaction mixture. After $3 \mathrm{~h}$, the solvent was evaporated under reduced pressure. Column chromatography $\left(\mathrm{CHCl}_{3}\right.$ :acetone:EtOH = 90:10:1 90:10:2) afforded the title compound (192 mg, 66\%); ${ }^{1} \mathrm{H}$ NMR (500 MHz, $\left.\mathrm{CDCl}_{3}\right) \delta 7.98(\mathrm{~m}, 1 \mathrm{H})$, 7.35-7.14 (m, 12H), $6.95(\mathrm{td}, J=16.5,2.5 \mathrm{~Hz}, 1 \mathrm{H}), 6.83(\mathrm{~m}, 1 \mathrm{H}), 5.12(\mathrm{dd}, J=44.5,12.5 \mathrm{~Hz}, 2 \mathrm{H}$, $\left.\mathrm{OC}_{2} \mathrm{Ph}\right), 4.89\left(\mathrm{q}, J=7.5 \mathrm{~Hz}, 1 \mathrm{H}, \underline{\mathrm{CHCH}}_{2} \mathrm{Ph}\right), 4.54\left(\mathrm{dd}, J=9.5,4.5 \mathrm{~Hz}, 1 \mathrm{H}, \mathrm{CHCO}_{2} \mathrm{Bn}\right), 3.74(\mathrm{~m}, 1 \mathrm{H}$, $\left.\mathrm{C}_{2} \mathrm{NR}_{2}\right), 3.63\left(\mathrm{~m}, 1 \mathrm{H}, \underline{\mathrm{C}}_{2} \mathrm{NR}_{2}\right), 3.18\left(\mathrm{dd}, J=9.0,5.0 \mathrm{~Hz}, 2 \mathrm{H}, \mathrm{CHC} \underline{H}_{2} \mathrm{Ph}\right), 2.93(\mathrm{~m}, 1 \mathrm{H}, \mathrm{C} \underline{\mathrm{HPr}}), 2.82$ (dd, $\left.J=16.5,9.5 \mathrm{~Hz}, 1 \mathrm{H}, \mathrm{CHCH}_{2} \mathrm{CO}_{2} \mathrm{NHR}\right), 2.47$ (dd, $\left.J=16.5,3.5 \mathrm{~Hz}, 1 \mathrm{H}, \mathrm{CHC}_{2} \mathrm{CO}_{2} \mathrm{NHR}\right), 2.16$ (m, 1H), $1,99(\mathrm{~m}, 3 \mathrm{H}), 1.59\left(\mathrm{~m}, 1 \mathrm{H}, \mathrm{CH}_{2} \mathrm{Et}\right), 1.38(\mathrm{~m}, 3 \mathrm{H}), 0.85\left(\mathrm{t}, J=7.3 \mathrm{~Hz}, 3 \mathrm{H}, \mathrm{CH}_{2} \mathrm{CH}_{3}\right) ;{ }^{13} \mathrm{C} \mathrm{NMR}(125$ $\left.\mathrm{MHz}, \mathrm{CDCl}_{3}\right) \delta 175.7,175.2,172.2,168.7,165.3(\mathrm{dd}, J=254.3,12.1 \mathrm{~Hz}, \underline{\mathrm{CF}}), 163.3,161.3(\mathrm{dd}, J=$ $252.5,12.1 \mathrm{~Hz}, \underline{\mathrm{CF}}), 136.1,135.8,133.7,129.5,128.9,128.8,128.5,128.4,127.3,116.8,112.5(\mathrm{~d}, J=20.4$ Hz), 104.6 (t, $J=27.0 \mathrm{~Hz}), 67.1,59.3,53.1,47.6$, 39.4, 38.4, 36.5, 34.5, 29.3, 25.0, 20.3, 14.3; HRMS (FAB) calcd for $\mathrm{C}_{35} \mathrm{H}_{38} \mathrm{~F}_{2} \mathrm{~N}_{3} \mathrm{O}_{7}\left(\mathrm{MH}^{+}\right)$650.2678, found 650.2676. 
(S)-2,4-Difluorobenzoyl-phenylalanine chloromethyl ketone (23). (S)-2,4-Difluorobenzoyl chloride $(3.88 \mathrm{~g}, 22 \mathrm{mmol})$ in dichloromethane $(36 \mathrm{~mL})$ was added to $24 \mathrm{~mL}$ of an aqueous solution of sodium carbonate $(3.18 \mathrm{~g}, 30 \mathrm{mmol})$, and then a solution of L-phenylalanine chloromethyl ketone $\mathbf{2 2}^{2}(4.68$ $\mathrm{g}, 20 \mathrm{mmol})$ in water $(40 \mathrm{~mL})$ was added dropwise with good stirring. After being stirred at room temperature for $5 \mathrm{~h}$, the solvent was removed under reduced pressure and the residue was purified by recrystallization from THF/hexane to give the title compound $(5.10 \mathrm{~g}, 76 \%) ;{ }^{1} \mathrm{H} \mathrm{NMR}\left(500 \mathrm{MHz}, \mathrm{CDCl}_{3}\right)$ $\delta 8.05(\mathrm{td}, J=8.5,6.5 \mathrm{~Hz}, 1 \mathrm{H}), 7.28(\mathrm{~m}, 5 \mathrm{H}), 7.16(\mathrm{dd}, J=12.0,6.0 \mathrm{~Hz}, 1 \mathrm{H}, \mathrm{NHCO}), 6.93(\mathrm{td}, J=8.3,2.5$ $\mathrm{Hz}, 1 \mathrm{H}), 6.86(\mathrm{ddd}, J=11.0,9.0,3.0 \mathrm{~Hz}, 1 \mathrm{H}), 5.12(\mathrm{qd}, J=7.0,1.5 \mathrm{~Hz}, 1 \mathrm{H}, \mathrm{RNHC} \underline{\mathrm{HCO}}), 4.14(\mathrm{dd}, J=$ 80.5, 16.0 Hz, 2H, $\underline{\mathrm{H}}_{2} \mathrm{Cl}$ ), 3.18 (ddd, $\left.J=35.5,13.5,7.0 \mathrm{~Hz}, 2 \mathrm{H}, \mathrm{CHC}_{2} \mathrm{Ph}\right) ;{ }^{13} \mathrm{C} \mathrm{NMR}(125 \mathrm{MHz}$, $\left.\mathrm{CDCl}_{3}\right) \delta 200.8,165.4(\mathrm{dd}, J=256.2,12.9 \mathrm{~Hz}, \underline{\mathrm{CF}}), 162.5(\mathrm{~d}, J=2.8 \mathrm{~Hz}$, ArCOONHR$), 161.4(\mathrm{dd}, J=$ 250.7, $12.9 \mathrm{~Hz}, \underline{\mathrm{CF}}), 135.5,134.0(\mathrm{dd}, J=10.2,3.6 \mathrm{~Hz}), 129.3,127.9,116.5(\mathrm{dd}, J=11.2,3.8 \mathrm{~Hz}, q)$, $112.8(\mathrm{dd}, J=21.4,2.8 \mathrm{~Hz}), 104.7(\mathrm{dd}, J=28.8,25.9 \mathrm{~Hz}), 58.2,47.8,37.7$; HRMS (FAB) calcd for $\mathrm{C}_{17} \mathrm{H}_{15} \mathrm{ClF}_{2} \mathrm{NO}_{2}\left(\mathrm{MH}^{+}\right)$338.0759, found 338.0741.

(S)-2,4-Difluorobenzoyl-phenylalanine acetoxymethyl ketone (24). This step was carried out according to a modified procedure of Remuzon. ${ }^{3}$ To a solution of $\mathbf{2 3}$ (4.32 g, $12.8 \mathrm{mmol}$ ) in dry DMF (30 $\mathrm{mL}$ ) under an argon atmosphere was added sodium iodide (1.90 g, $12.7 \mathrm{mmol})$, and potassium acetate $(2.49 \mathrm{~g}, 25.4 \mathrm{mmol})$. The reaction mixture was stirred at room temperature for $17 \mathrm{~h}$, and poured into saturated aqueous $\mathrm{NH}_{4} \mathrm{Cl}(40 \mathrm{~mL})$. The mixture was extracted with EtOAc $(100 \mathrm{~mL} \times 2)$. The combined organic layer was washed with brine $(60 \mathrm{~mL} \times 3)$, dried over $\mathrm{MgSO}_{4}$, filtered and was concentrated under reduced pressure to give $24(4.57 \mathrm{~g}, 99 \%)$ as an oil; ${ }^{1} \mathrm{H} \mathrm{NMR}\left(500 \mathrm{MHz}, \mathrm{CDCl}_{3}\right) \delta 8.06(\mathrm{td}, J=9.0,7.0$ $\mathrm{Hz}, 1 \mathrm{H}), 7.28(\mathrm{~m}, 5 \mathrm{H}), 7.14(\mathrm{dd}, J=12.0,6.5 \mathrm{~Hz}, 1 \mathrm{H}, \mathrm{NHCO}), 6.98(\mathrm{td}, J=8.3,2.5 \mathrm{~Hz}, 1 \mathrm{H}), 6.85$ (ddd, $J$ $=11.5,8.5,2.5 \mathrm{~Hz}, 1 \mathrm{H}), 5.05(\mathrm{qd}, J=7.0,1.5 \mathrm{~Hz}, 1 \mathrm{H}, \mathrm{RNHC} \underline{\mathrm{HCO}}), 4.73(\mathrm{dd}, J=37.5,17.0 \mathrm{~Hz}, 2 \mathrm{H}$, $\left.\mathrm{C}_{2} \mathrm{OAc}\right), 3.17$ (ddd, $\left.J=45.5,14.0,7.0 \mathrm{~Hz}, 2 \mathrm{H}, \mathrm{CHC}_{2} \mathrm{Ph}\right), 2.16\left(\mathrm{~s}, 3 \mathrm{H}, \mathrm{COC}_{3}\right) ;{ }^{13} \mathrm{C} \mathrm{NMR}(125 \mathrm{MHz}$, $\left.\mathrm{CDCl}_{3}\right) \delta 202.1,170.4,165.4(\mathrm{dd}, J=256.2,12.9 \mathrm{~Hz}, \underline{\mathrm{CF}}), 162.5$ (d, $J=2.8 \mathrm{~Hz}$, ArCONHR), 161.4 (dd, $J$ $=250.7,12.9 \mathrm{~Hz}, \underline{\mathrm{CF}}), 135.6,134.0(\mathrm{dd}, J=10.2,3.8 \mathrm{~Hz}), 129.5,129.2,127.7,116.7(\mathrm{dd}, J=11.2,3.8 \mathrm{~Hz}$, q), $112.7(\mathrm{dd}, J=21.4,2.8 \mathrm{~Hz}), 104.7(\mathrm{dd}, J=28.8,25.9 \mathrm{~Hz}), 67.3,57.5,37.4,20.6$; HRMS (FAB) calcd for $\mathrm{C}_{19} \mathrm{H}_{18} \mathrm{~F}_{2} \mathrm{NO}_{4}\left(\mathrm{MH}^{+}\right)$362.1204, found 362.1210.

(S)-2,4-Difluorobenzoyl-phenylalanine hydroxymethyl ketone (25). A modified procedure of Mori $^{4,5}$ was applied. KCN (100 mg, $\left.1.5 \mathrm{mmol}\right)$ was added to a solution of $24(2.00 \mathrm{~g}, 5.5 \mathrm{mmol})$ in 
MeOH:THF: $\mathrm{H}_{2} \mathrm{O}(8: 2: 1,33 \mathrm{~mL})$ and the resultant solution was stirred at room temperature overnight. The solvent was evaporated under reduced pressure and the residue was taken up in dichloromethane. The organic layer was separated, dried over $\mathrm{MgSO}_{4}$, filtered and concentrated under reduced pressure. Column chromatography (hexane:EtOAc $=3: 2 \sim 1: 1)$ afforded the title compound $(1.41 \mathrm{~g}, 80 \%) ;{ }^{1} \mathrm{H}$ NMR $(500$ $\left.\mathrm{MHz} \mathrm{CDCl}_{3}\right) \delta 8.04(\mathrm{td}, J=8.5,6.5 \mathrm{~Hz}, 1 \mathrm{H}), 7.26(\mathrm{~m}, 5 \mathrm{H}), 7.13(\mathrm{dd}, J=12.0,6.5 \mathrm{~Hz}, 1 \mathrm{H}, \mathrm{NHCO}), 6.97$ $(\mathrm{td}, J=8.0,2.5 \mathrm{~Hz}, 1 \mathrm{H}), 6.85(\mathrm{ddd}, J=11.0,9.0,3.0 \mathrm{~Hz}, 1 \mathrm{H}), 5.04(\mathrm{qd}, J=7.0,1.5 \mathrm{~Hz}, 1 \mathrm{H}, \mathrm{RNHC} \underline{\mathrm{HCO}})$, $4.26\left(\mathrm{dd}, J=67.0,19.5 \mathrm{~Hz}, 2 \mathrm{H}, \underline{\mathrm{C}}_{2} \mathrm{OH}\right), 3.16\left(\mathrm{qd}, J=14.0,7.0 \mathrm{~Hz}, 2 \mathrm{H}, \mathrm{CHC}_{2} \mathrm{Ph}\right), 2.78(\mathrm{bs}, 1 \mathrm{H}, \mathrm{OH})$; ${ }^{13} \mathrm{C}$ NMR $\left(125 \mathrm{MHz}, \mathrm{CDCl}_{3}\right) \delta 208.7,165.3(\mathrm{dd}, J=256.2,12.9 \mathrm{~Hz}, \underline{\mathrm{CF}}), 162.6(\mathrm{~d}, J=2.9 \mathrm{~Hz}$, ArCONHR), 161.3 (dd, $J=250.7,12.1 \mathrm{~Hz}, \underline{\mathrm{C} F}), 135.6,133.9$ (dd, $J=10.2,2.8 \mathrm{~Hz}), 129.3,129.2,127.7$, $116.8(\mathrm{dd}, J=12.1,3.8 \mathrm{~Hz}, q), 112.7(\mathrm{dd}, J=21.4,3.8 \mathrm{~Hz}), 104.6(\mathrm{dd}, J=28.8,26.0 \mathrm{~Hz}), 67.7,57.2,37.5$; HRMS (FAB) calcd for $\mathrm{C}_{17} \mathrm{H}_{16} \mathrm{~F}_{2} \mathrm{NO}_{3}\left(\mathrm{MH}^{+}\right)$320.1098, found 320.1112.

\section{1-(2(R)-\{(S)-Acetoxy-[3(S)-(2,4-difluoro-benzoylamino)-2-oxo-4-phenyl-butoxycarbonyl]-}

methyl\}-pentanoyl)-proline benzyl ester (17). Diisopropylethylamine (280 mL, $1.6 \mathrm{mmol})$ was added portionwise to a solution of acid $11(460 \mathrm{mg}, 0.95 \mathrm{mmol})$ and hydroxymethyl ketone 25 (275 $\mathrm{mg}, 0.86$ $\mathrm{mmol})$ in DMF (4 mL) in the presence of PYBOP $(445 \mathrm{mg}, 0.85 \mathrm{mmol})$ at $-10{ }^{\circ} \mathrm{C}$. The resultant solution was stirred at $4{ }^{\circ} \mathrm{C}$ overnight. An additional amount of diisopropylethylamine $(52 \mathrm{~mL}, 0.30 \mathrm{mmol})$ was added to the reaction mixture. After an additional $8 \mathrm{~h}$, the solvent was evaporated under reduced pressure. Column chromatography $\left(\mathrm{CHCl}_{3}: \mathrm{EtOH}: \mathrm{AcOH}=60: 1: 0.1\right)$ afforded the title compound (313 mg, 52\%); ${ }^{1} \mathrm{H}$ NMR $\left(500 \mathrm{MHz}, \mathrm{CDCl}_{3}\right) \delta 8.04(\mathrm{~m}, 1 \mathrm{H}), 7.29(\mathrm{~m}, 10 \mathrm{H}), 7.11(\mathrm{dd}, J=12.0,6.5 \mathrm{~Hz}, 1 \mathrm{H}), 6.97(\mathrm{td}, J=$ 8.3, 2.5 Hz, 1H), $6.84(\mathrm{ddd}, J=11.5,8.5,2.5 \mathrm{~Hz}, 1 \mathrm{H}), 5.16\left(\mathrm{q}, J=13.5 \mathrm{~Hz}, 2 \mathrm{H}, \mathrm{OC}_{2} \mathrm{Ph}\right), 5.06(\mathrm{dd}, J=$ 10.0, $5.5 \mathrm{~Hz}, 1 \mathrm{H}, \mathrm{CHOAc}), 4.96$ (m, 1H, RNHCㅂC), 4.82 (dd, $J=58.5,17.0 \mathrm{~Hz}, 2 \mathrm{H}, \mathrm{COCH}_{2} \mathrm{O}_{2} \mathrm{CR}$ ), $4.62\left(\mathrm{dd}, J=9.0,4.5 \mathrm{~Hz}, 1 \mathrm{H}, \mathrm{C}_{\underline{H} C O} \mathrm{Bn}\right), 3.77\left(\mathrm{~m}, 1 \mathrm{H}, \underline{\mathrm{C}}_{2} \mathrm{NR}_{2}\right), 3.69\left(\mathrm{~m}, 1 \mathrm{H}, \mathrm{C}_{2} \mathrm{NR}_{2}\right), 3.23$ (dd, $J=$ 14.5, $\left.6.5 \mathrm{~Hz}, 1 \mathrm{H}, \mathrm{CHC}_{2} \mathrm{Ph}\right), 3.13(\mathrm{~m}, 2 \mathrm{H}), 2.21\left(\mathrm{~m}, 1 \mathrm{H}, \underline{\mathrm{C}}_{2} \mathrm{CHCO}_{2} \mathrm{Bn}\right), 2.04$ (s, 3H, COC$\left.\underline{H}_{3}\right), 2.07-$ $1.95(\mathrm{~m}, 3 \mathrm{H}), 1.77\left(\mathrm{~m}, 1 \mathrm{H}, \mathrm{CH}_{2} \mathrm{Et}\right), 1.66\left(\mathrm{~m}, 1 \mathrm{H}, \underline{\mathrm{CH}}_{2} \mathrm{Et}\right), 1.49\left(\mathrm{~m}, 1 \mathrm{H}, \mathrm{CH}_{2} \mathrm{CH}_{3}\right), 1.28\left(\mathrm{~m}, 1 \mathrm{H}, \mathrm{CH}_{2} \mathrm{CH}_{3}\right)$, $0.88\left(\mathrm{t}, J=7.3 \mathrm{~Hz}, 3 \mathrm{H}, \mathrm{CH}_{2} \underline{\mathrm{C}}_{3}\right) ;{ }^{13} \mathrm{C} \mathrm{NMR}\left(125 \mathrm{MHz}, \mathrm{CDCl}_{3}\right) \delta 201.1,172.0,170.9,170.2(\mathrm{~d}, J=3.9$ $\mathrm{Hz}), 169.4,165.4(\mathrm{dd}, J=256.2,12.9 \mathrm{~Hz}, \underline{\mathrm{CF}}), 162.6,161.4(\mathrm{dd}, J=250.7,12.1 \mathrm{~Hz}, \underline{\mathrm{C}} \mathrm{F}), 135.9,135.7$, $134.0(\mathrm{dd}, J=10.2,3.8 \mathrm{~Hz}), 129.4,129.2,128.8,128.5,128.4,127.8,116.6(\mathrm{dd}, J=12.1,3.8 \mathrm{~Hz}, q), 112.7$ $(\mathrm{dd}, J=21.4,2.8 \mathrm{~Hz}), 104.7(\mathrm{dd}, J=27.9,26.0 \mathrm{~Hz}), 74.5,67.8,67.1,59.2,57.8,47.7,44.9,37.0,30.5$, 29.6, 25.2, 20.7, 20.0, 14.4; HRMS (FAB) calcd for $\mathrm{C}_{38} \mathrm{H}_{41} \mathrm{~F}_{2} \mathrm{~N}_{2} \mathrm{O}_{9}\left(\mathrm{MH}^{+}\right)$707.2780, found 707.2786. 


\section{References}

(1) Simoneau, B.; Lavallée, P.; Anderson, P. C.; Bailey, M.; Bantle, G.; Berthiaume, S.; Chabot, C.; Fazal, G.; Halmos, T.; Ogilvie, W. W.; Poupart, M.-A.; Thavonekham, B.; Xin, Z.; Thibeault, D.; Bolger, G.; Panzenbeck, M.; Winquist, R.; Jung, G. L. Bioorg. Med. Chem. 1999, 7, 489-508.

(2) Onishi, T.; Nakano, T.; Hirose, N.; Nakazawa, M.; Izawa, K. Tetrahedron Lett. 2001, 42, 5887 5890.

(3) Remuzon, P.; Dussy, C.; Jacquet, J.-P.; Roty, P.; Bouzard, D. Tetrahedron Asymm. 1996, 7, 1181-1188.

(4) Mori, K.; Tominaga, M.; Takigawa, T.; Matsui, M. Synthesis 1973, 790-791.

(5) Mori, K.; Sasaki, M. Tetrahedron Lett. 1979, 42, 1329-1332. 


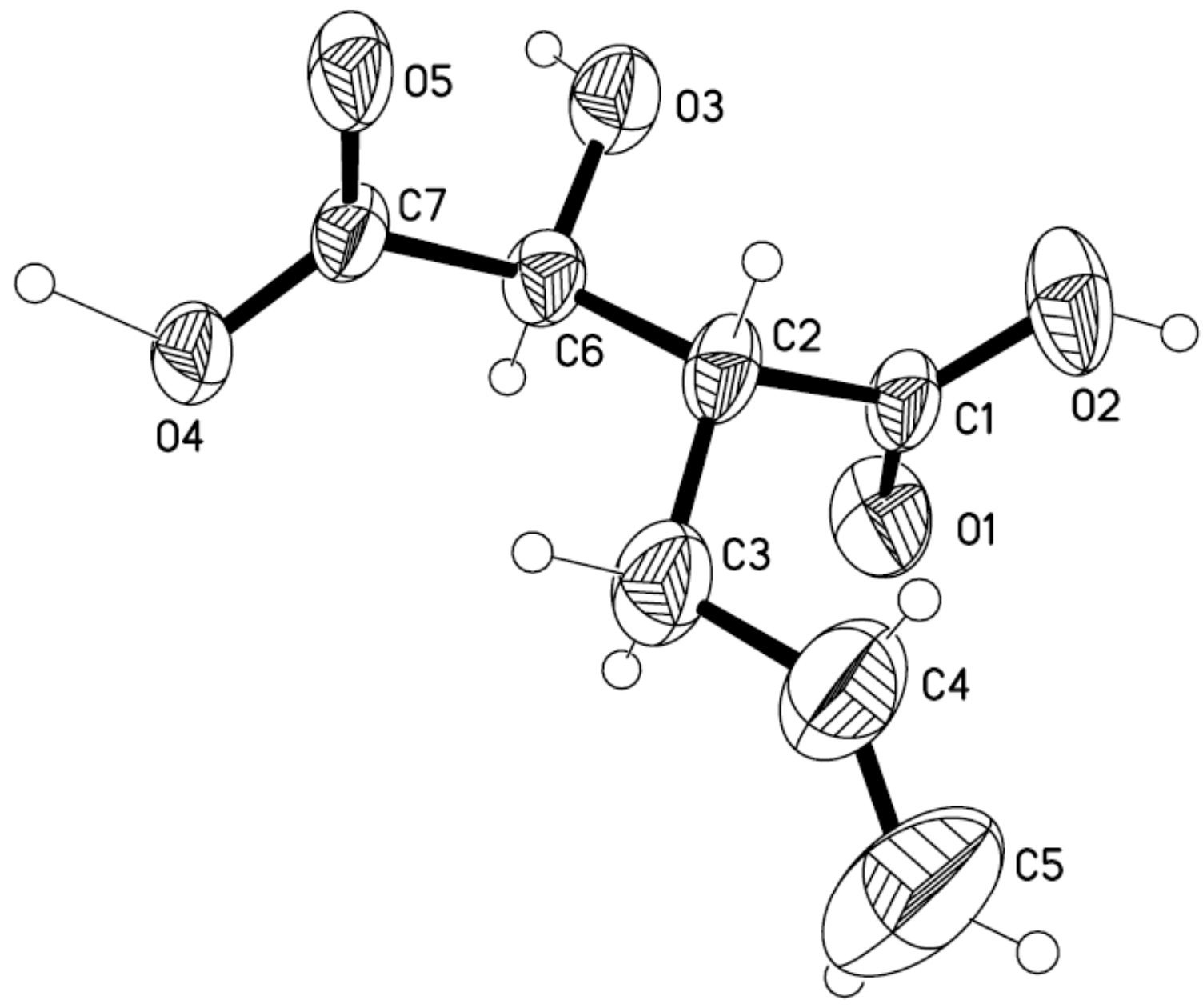

Figure S1. Crystal structure of compound 6, (2R,3S)-diisopropyl 2-allyl-3-hydroxysuccinate. 